Journal of Engineering and Applied Sciences 14 (13): 4547-4552, 2019

ISSN: 1816-949X

(C) Medwell Journals, 2019

\title{
Electronic System for Detection and Measurement of Water Turbidity by Means of Artificial Vision
}

\author{
J. Vargas, J. Guapacho and A. Cucaita \\ Macrypt Research Group, Eysi Study Group, Faculty of Basic Sciences and Engineering, \\ University of Llanos, Villavicencio, Colombia
}

\begin{abstract}
The study presents the development of an electronic system for the detection and measurement of water turbidity using artificial vision. Turbidity is one of the characteristics of water that allows its quality to be determined, it facilitates decision-making about the treatment to be received or whether, it is suitable for human consumption. The importance of this parameter has led to the development and improvement of techniques and instruments to measure the level of turbidity of water. The objective of this research is to use artificial vision techniques evaluate. The mathematical model implemented presents errors of $5 \%$ with respect to the value taken by the standard equipment to determine the level of turbidity of different water samples. The implanted artificial vision system consists of different phases in which digital image processing and analysis techniques are combined.
\end{abstract}

Key words: Image recognition, turbidity, artificial vision, image processing, image analysis, analysis techniques

\section{INTRODUCTION}

The universe can be interpreted through the different variables that compose it the human being interprets some of these variables through his senses, allowing it to make decisions and carry out acciones. Electronics from the field of instrumentation is able to accurately interpret variables in different systems to automatically perform actions that control the behavior of a system. Literature papers showing processes of control and creation of measuring instruments can be found (Sultana et al., 2018; Guativa et al., 2015, 2016).

The quality of water is a factor of great implications for human health and its use in different industries for what has been different techniques and instruments have been implemented to measure the quality of this (Kazemian et al., 2017). There are different parameters that make it possible to determine the quality of the water, these parameters are turbidity, colour, total solids, filterable solids (dissolved), dissolved oxygen, $\mathrm{pH}$, Chemical Oxygen Demand (COD). Ammoniacal nitrogen, phosphates and waste water toxins for the measurement of these parameters various laboratory techniques are applied which allow their characterization. Turbidity is defined by the International Organization for Standardization (ISO) as the reduction of the transparency of a liquid caused by the presence of undissolved particles of material other than the liquid itself (Acebo-Gonzalez and Hernandez-Garcia, 2013). There are different units and methods for measuring turbidity such as the visual (Jackson Spark Plug Turbidimeter (JTU)) or light scattering method, the hach turbidimeter or a spectrophotometer with nephelometric equipment (attached device for measuring scattered light). In the literature and in laboratory tests, they are used with devices and techniques that use 'Nephelometric turbidity units' or 'NTUs' as a reference, these measurements are standardized with a standard suspension of the polymer formacin (Sultana et al., 2018). Water with enough suspended clay particles (10 units of turbidity) can be seen with the naked eye, surface water sources vary from 10-1,000 units of turbidity and very opaque rivers can reach 10,000 units (Rodriguez, 2014; Vargas et al., 2018; Zain et al., 2017).

Other way in which it is possible to determine water turbidity is through image analysis and artificial vision techniques, the purpose of image analysis is to quantify the properties of the objects present in the images (Lira, 2010). This process makes it possible to obtain a measure, interpretation or decision from an image (Mery, 2004). In Digital Image Processing (DIP), an image is taken and a modified version of the image is produced. It is widely used in different applications in robotics, communications, remote sensing, biomedicine, industrial automation, inspection systems, navigation, optical measurements, among others (Mery, 2004; Lopez et al., 2015).

Image processing and analysis techniques are widely used to determine water quality in large bodies of water using satellite imagery (Polvorinos et al., 2001). On a

Corresponding Author: J. Vargas, Macrypt Research Group, Eysi Study Group, Faculty of Basic Sciences and Engineering, University of Llanos, Villavicencio, Colombia 
smaller scale, digital cameras and characterizations are used to formulate a mathematical model to determine the turbidity of water samples (Karnawat and Patil, 2016).

In the case of this research, the python programming language and the open $\mathrm{CV}$ artificial vision library were used. Unlike, most programming languages, python provides style rules, so, you can write more readable source code in a standardized way (Bahit, 2012).

\section{MATERIALS AND METHODS}

For the implementation of this research, a methodology oriented to prototypes by stages was used. Each stage contributed to image processing, analysis and interpretation. The phases used were image acquisition and pre-processing, segmentation, processing, measurement and interpretation.

Image acquisition and pre-processing: The acquisition of the image was made by means of a webcam, this camera allowed a real time analysis of the sample and the capture of images with a size of $640 \times 480$.

The captured images were in RGB (Red-Green-Blue) format. The artificial vision system implemented required segmentation to identify and isolate the turbid water sample or segments of it from the image. Variations in the light or position from which them image was captured meant that the RGB format with which the images were initially captured was not the most appropriate for which reason a conversion to HSV format was made. The HSV format decomposes the colors of the image into its Hue, saturation, value luminance, a more natural way of describing colors, for example, by allowing the value/luminance component to be discarded, making the algorithm less sensitive to light conditions in the input image.

Segmentation: The segmentation stage was implemented to identify and differentiate the elements present in the image, the background and the sample. The transformation of the images to the HSV format allowed the identification process to be precise in the identification of the background color for this purpose the values of the HSV bands were varied until the optimum value range was found. These values made it possible to identify the background color of the image and therefore the geometric shape of the water container.

The identification of the background resulted in a mask consisting of a matrix containing values of 0 or 255 where the pixels with 255 corresponded to the background and were observed in white and the pixels with a value of 0 corresponded to the container containing the sample and were observed in black.
Processing: The segmentation allowed to obtain an image of the object of analysis which is called a mask. In order to improve the image quality of the mask, processing using computer algorithms and digital filters is necessary. The closing and opening filters were used to eliminate the noise that was presented in the form of small points, these filters base their operation on the mixture of the morphological operators, dilation and erosion.

In the morphological erosion operator an object is uniformly reduced in its spatial extent. Suppose that object $\mathrm{X}$ and structural element $\mathrm{B}$ are represented as sets in a two-dimensional Euclidean space and $\mathrm{Bx}$ is the translation of $\mathrm{B}$, so that, its origin is located in $\mathrm{x}$, then the erosion of $\mathrm{X}$ by $\mathrm{B}$ is defined as the set of points (pixels) $\mathrm{x}$ such that $\mathrm{Bx}$ is contained in $\mathrm{X}$, symbolically Eq. 1 :

$$
\varepsilon \mathrm{B}=\mathrm{X} \ominus \mathrm{B}=\{\mathrm{x} \mid \mathrm{Bx} \subseteq \mathrm{X}\}=\{\mathrm{x} \mid \mathrm{B}+\mathrm{x} \subseteq \mathrm{X}\}
$$

In the morphological operator dilation an object grows uniformly in its spatial extension, this by means of the path of a component through the pixels of the elements contained in a matrix. Similar to erosion, the expansion of $\mathrm{X}$ by $\mathrm{B}$ is defined as the set of $\mathrm{x}$-points such that B intersects X, symbolically Eq. 2:

$$
\varepsilon \mathrm{B}=\mathrm{X} \ominus \mathrm{B}=\{\mathrm{x} \mid \mathrm{Bx} \subseteq \mathrm{X}\}=\{\mathrm{x} \mid \mathrm{B}+\mathrm{x} \subseteq \mathrm{X}\}
$$

The closing filter, obtained after applying an image dilation followed by erosion is useful to eliminate small holes (dark regions). On the other hand, opening is obtained by the erosion of an image followed by dilation which is useful for removing small objects (objects are light points in black foreground Algorithm 1:

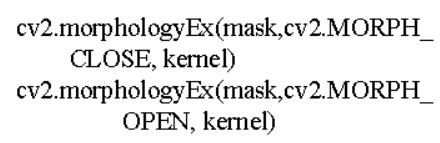

(AL.1)

The lines of code presented in Algorithm 1 correspond to the code used in the python programming language and using the OpenCV library where "mask" corresponds to the mask to be worked on and "kernel" refers to the structural element that passes through the object.

The Gaussian filter was used to smooth the edges of the detected segment. This filter allows the noise attenuation to be less aggressive at the edges due to the shape of its function and results from the application of a filter mask defined by a two-dimensional Gaussian function Eq. 3: 


$$
\mathrm{G}(\mathrm{x}, \mathrm{y})=\frac{1}{\sqrt{2 \pi \sigma}} \mathrm{e}^{-\frac{\mathrm{x}^{2}+\mathrm{y}^{2}}{2 \sigma^{2}}}
$$

The lines correspond to the code used in the pyhon programming language and using the OpenCV library, where "mask" is the image you want to work on, the following factor" $(5,5)$ " indicates the use of the $5 \times 5 \mathrm{kernel}$ and the last factor " 0 " corresponds to the standard deviation in $\mathrm{X}$ and $\mathrm{Y}$ being " 0 " calculated from the size of the matrix Algorithm 2:

\begin{tabular}{|l|l|}
\hline cv2.GaussianBlur(mask, $(5,5), 0)$ & (AL.2) \\
\hline
\end{tabular}

It was necessary to detect the edges of the sample or the sample segment, thus, facilitating the capture of the sector that is required for this task, the canny algorithm was used, ideal for its low error rate in edge detection. Canny's algorithm can be divided into three major steps: Obtaining the gradient: this step starts by applying a Gaussian filter and ends by calculating the magnitude and orientation of the gradient vector in each pixel. The gradient of an image $f(x, y)$ at the point $(x, y)$ is defined by a two-dimensional vector given by Eq. 4:

$$
G[f(x, y)]=\left[\begin{array}{l}
G_{x} \\
G_{y}
\end{array}\right]=\left[\begin{array}{l}
\frac{\partial}{\partial x} f(x, y) \\
\frac{\partial}{\partial y} f(x, y)
\end{array}\right]
$$

where, the vector $\mathrm{G}$ points in the direction of maximum variation of $f$ at the point $(x, y)$ per unit distance with the magnitude and direction given by:

$$
\begin{aligned}
& |\mathrm{G}|=\sqrt{\mathrm{G}_{\mathrm{x}}^{2}+\mathrm{G}_{\mathrm{y}}^{2}}=\left|\mathrm{G}_{\mathrm{x}}\right|+\left|\mathrm{G}_{\mathrm{y}}\right| \\
& \varphi(\mathrm{x}, \mathrm{y})=\tan ^{-1} \frac{\mathrm{G}_{\mathrm{y}}}{\mathrm{G}_{\mathrm{x}}}
\end{aligned}
$$

No maximum suppression: in this step, we achieve the thinning of the width of the edges obtained with the gradient, eliminating the pixels that are not considered part of the border and obtaining as a result only thin lines. Threshold hysteresis: a hysteresis function based on two thresholds (upper and lower) is applied, this process is intended to reduce the possibility of false contours (Rebaza, 2007) Algorithm 3:

\begin{tabular}{l|l} 
cv2.Canny(Image, Low, Up) & (AL.3)
\end{tabular}

The previous line corresponds to the code used in the pyhon programming language using the OpenCV library where the image refers to the image to which the edges will be detected, "Low" and "Up" refers to the lower and upper values with which the threshold hysteresis is performed.

Sector recovery with the sample: After the filters were applied, the matrix of the original image was scrolled through in each of the three bands that make up the RGB (Red, Green, Blue) and an "or" operation was performed between each pixel and its corresponding position in the mask. The "or" operation involved a transformation in the image as each of the pixels that did not correspond to the container with the sample, once the mask was applied, obtained a value of 255 . This caused the resulting image to be white in all places that do not correspond to the sample.

Measurement: The RGB color format was converted. The pixels, went from being represented by 3 different values, to having a single value that corresponded to a value in a gray scale made up of 256 levels which vary between black (0) and white (255).

The measurements were made using the histogram of the images, a histogram consists of a representation of the weights of each of the pixels. The process to find the weights of the pixels was done by means of a representation of the image in gray scale. This way it can be calculated that, so many, pixels were found in each of the grey levels.

Interpretation: The interpretation allowed to know the approximate turbidity value, this was carried out by means of the application of a mathematical model which described the relationship between the input (gray level with more weight) and output (value in NTU). The mathematical model implemented corresponded to a second order polynomial approximation in the same way, different samples were used which were subjected to a laboratory study that allowed the identification of turbidity level in NTU.X.

\section{RESULTS AND DISCUSSION}

Image processing: The filters and algorithms applied are responsible for making changes in the image or mask, these changes can be seen below. Figure 1, refers to the image that was originally captured and corresponds to a $640 \times 480$ size image.

Figure 2, corresponds to the result of identifying the background and the sample, however, small areas were also observed that were not part of the sample. The processing of the images began with the application of the closing and opening filters. The result of this can be seen in Fig. 3, these filters eliminated the noise that appeared in the form of small black and white spots. 


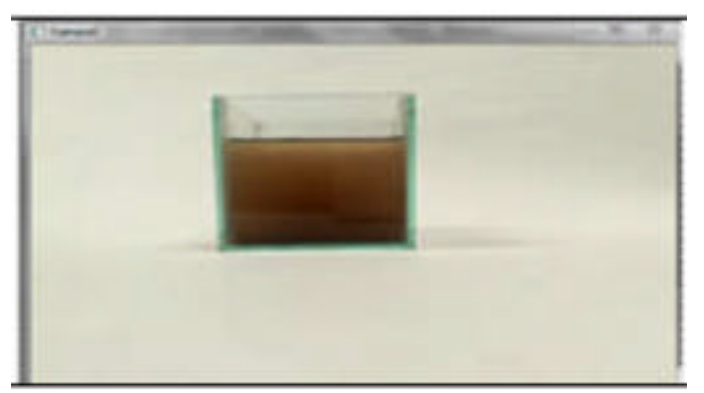

Fig. 1: Image acquisition

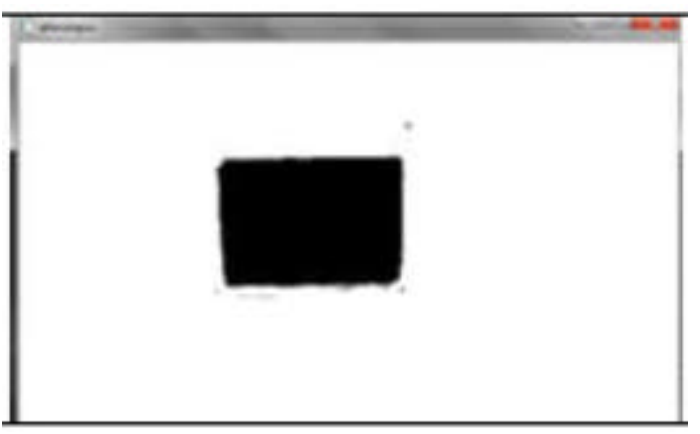

Fig. 2: Segmentation (background identification)

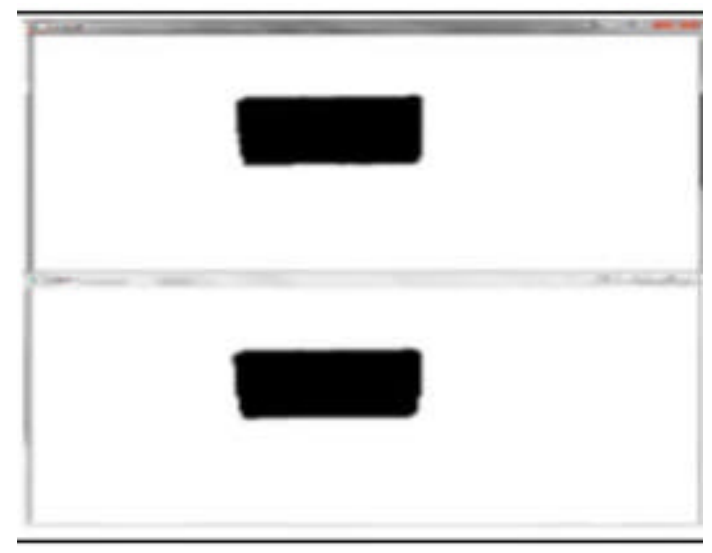

Fig. 3: Segmentation (background identification)

After the application of the closing and opening filters, the Canny algorithm was used for which it was first necessary to apply a Gaussian filter which resulted in Fig. 4 where the edges of the sample are slightly blurred. After the Gaussian filter was applied the line of code that allowed the Canny algorithm to be put into practice, thus, detecting the edges of the sample as shown in Fig. 4 and 5 .

The detected edges were superimposed on the original image as shown in Fig. 6 in order to allow the operator to know the area or segment to be captured and on which the subsequent analysis would be performed.

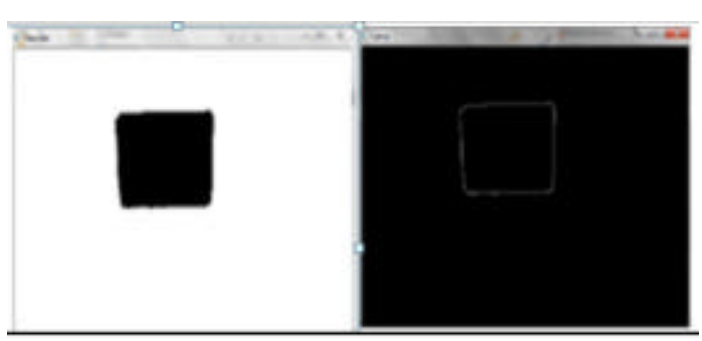

Fig. 4: Gaussian filter and Canny algorithm

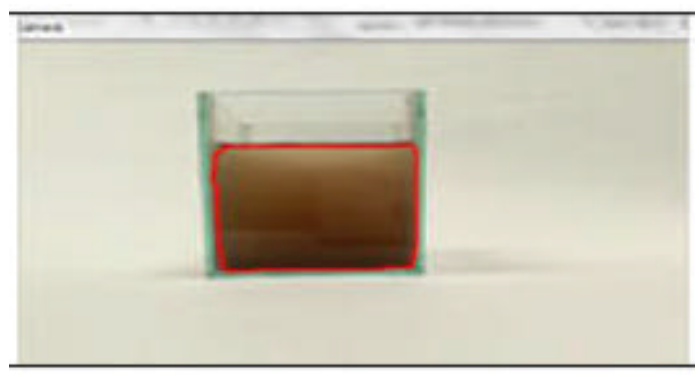

Fig. 5: Sector identifed

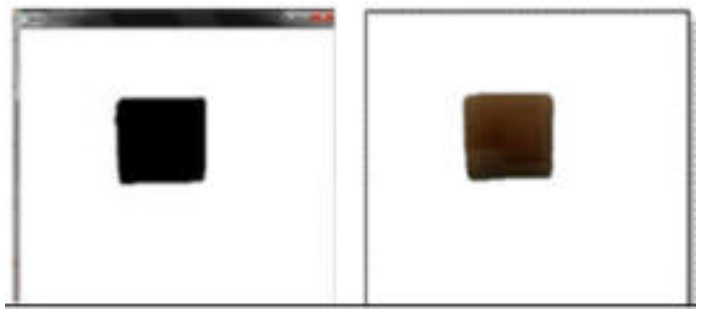

Fig. 6: Image processing

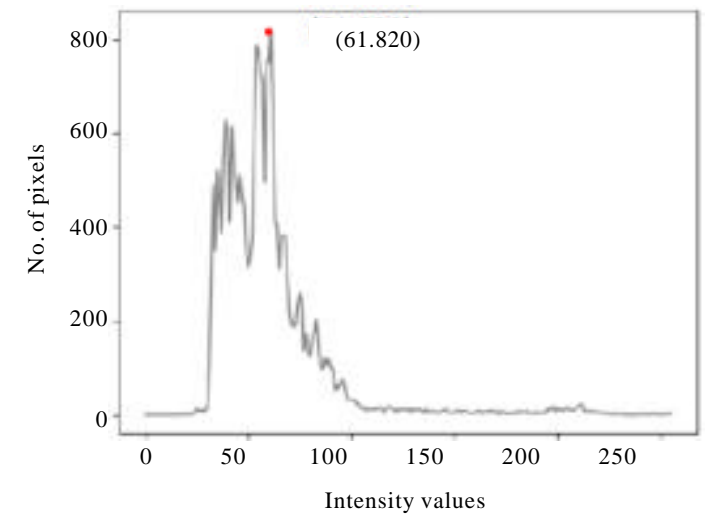

Fig. 7: Histogram number of pixels vs. intensity light

The mask or image that was obtained as a result after the application of filters is shown in Fig. 7. Figure 6 it is possible to see the final result of the image processing where only the sample containing the turbid water was recovered. 
Table 1: Turbidity and gray levels

\begin{tabular}{lc}
\hline Turbidity level in NTU, laboratory tests & Gray scale most often on image \\
\hline 172 & 61 \\
156.5 & 96 \\
121 & 125 \\
72.5 & 158 \\
39 & 173 \\
\hline
\end{tabular}

Table 2: Laboratory value, mathematical model values and error (\%)

\begin{tabular}{lcc}
\hline Real NTU & NTU mathematical model & Error $(\%)$ \\
\hline 172 & 172.5 & 0.29 \\
156.5 & 155 & 1.06 \\
121 & 124 & 2.5 \\
72.5 & 70 & 2.5 \\
39 & 40 & 4 \\
\hline
\end{tabular}

Image analysis and mathematical modeling: Once the treatment of the images was carried out which allowed to isolate the turbid water sample or sectors of it, we proceeded with the analysis of the same, using the histogram for this task. The data from the histogram allows to determine the different shades of gray present in the image and the number of pixels present in this shade.

Figure 7 shows the histogram of a sample with a turbidity value of 172 NTU and shows different grey levels, 61 being the most repeated grey level. The grey level that was most repeated in each of the samples was the one taken as a reference to formulate the mathematical model.

The gray level with the largest number of pixels or weight, varied in the gray scale approaching the value $O$ (black) as the sample increased the level of turbidity as shown in Table 1, likewise different levels of gray were observed, this was due to the material from which the container was built, the reflection that could be generated on it and that the turbidity varied in a non-uniform way in the sample, causing different levels of turbidity to exist in the same

The mathematical model carried out was a second order polynomial representation, capable of representing the relationship between the grey level most frequently obtained and the results obtained in the laboratory of the level of turbidity of the samples:

$$
\mathrm{Y}=-0.0088 \mathrm{X}^{2}+0.8796 \mathrm{X}+151.59
$$

where, $\mathrm{Y}$ is the value given by Eq. 6 and corresponds to the approximate turbidity in NTU and X refers to the gray level that has the most pixels or weight.

To check the level of error between the model and the actual values, calculations were made which allowed approximate NTU values to be obtained and a comparison to be made with the values measured in the laboratory, resulting in an error of $<5 \%$ (Table 2 ).
Table 3: Tests with different levels of sediment

\begin{tabular}{lrrrr}
\hline Levels & Sample 1 & Sample 2 & Sample 3 & Sample 4 \\
\hline Gray level with higher weight & 138 & 109 & 94 & 71 \\
Water (\%) & 99.84 & 99.60 & 99.21 & 98.81 \\
Sediment (\%) & 0.16 & 0.40 & 0.79 & 1.19 \\
NTU Mathematical model & 105.39 & 142.91 & 156.52 & 169.68 \\
\hline
\end{tabular}

Verification: In order to check the functioning of the designed model, different tests were carried out where the percentage of sediments in the samples varied (The sediment used was the same type of sediment used for the mathematical model formulation). This verification allowed to know the approximate value of the turbidity of the sample as well as to corroborate that as the percentage of sediments increased, so did the turbidity measurement in NTU, Table 3.

Water is an indispensable resource for human life, its characteristics and composition, directly affects the quality of life of the human being. Turbidity is a parameter that allows us to determine and have a vision of water quality, so, it is essential to develop methodologies or techniques to determine the level of turbidity accurately and economically.

In the development of this research a process of image processing and analysis was carried out, to formulate a mathematical model to allow the water's turbidity value to be measured. Some research comparing (Polvorinos et al., 2001; Karnawat and Patil, 2016; Mizutani, 1993). In these by means of image processing and analysis it was explained how it was possible to make approximate measurements of the turbidity level in large bodies of water or laboratory samples, using satellite images or images captured by digital cameras. All of this is done by means of a characterization in which measurement instruments are used to determine the real turbidity value. Some of the consulted works resorted to the use of histograms, to assign a characteristic value to the images and thus, to generate a mathematical model or a data base with which the turbidity value of future samples can be known. In other research, mathematical models are formulated from the interpretation of the variation in each of the color bands that make up the image in RGB format. With the work done it can be determined that using these methodologies it is possible to measure turbidity level using artificial vision, provided that the environmental characteristics, the type of sediment in the sample and the reflection of the container are taken into account, abrupt changes in these variables caused that the mathematical model proposed did not work properly. In addition, it was found that for a low level of turbidity $(<30 \mathrm{NTU})$, measurements were not possible. 


\section{CONCLUSION}

When using the grey levels of an image to formulate a mathematical model that describes the variation in the level of turbidity, a good level of correspondence was found between the model and the real values where the error does not exceed $5 \%$.

Variables such as light, type of sediment or reflection play a key role in image processing and analysis and must, therefore, be taken into account, so that, once a mathematical model has been formulated, the measurements present a low percentage of error.

The segmentation of the images generated unwanted segments, the closing and opening filters allowed to eliminate the unwanted segments, thus, isolating the desired sector, the image that contained the sample.

The canny algorithm, allowed to detect the edges of the sample that was segmented in the image, helping in a significant way, since, it allowed to know which would be the sector that later would analyze and the area of this one.

In the analysis of images, the histogram is a simple tool, however, for this research, it allowed to extract from the image the necessary information that allowed to obtain the approximate value of the level of turbidity of water contaminated with sediments.

\section{REFERENCES}

Acebo-Gonzalez, D. and A.T. Hernandez-Garcia, 2013. [Turbidimetric methods and their applications in the life sciences (In Spanish)]. CENIC. Mag. Biol. Sci., 44: $1-18$.

Bahit, E., 2012. [Python Course for Beginners]. Eugeniabahit.Com, Murcia, Spain, Pages: 136 (In Galician).

Guativa, J.A.V., C.J.A. Leon, G. Castro and J. James, 2016. [Virtual instrumentation system using an experimental RTD sensor (In Galician)]. Ingenium, 17: 31-46.

Guativa, J.A.V., J.A.L. Velasquez andL.C. Cardenas, 2015. [Instrumentation and control system for drinking water storage tanks (In Spanish)]. Ingeniare, 17: 69-85.

Karnawat, V. and S.L. Patil, 2016. Turbidity detection using image processing. Proceedings of the 2016 International Conference on Computing, Communication and Automation (ICCCA), April 29-30, 2016, IEEE, Noida, India, ISBN: 978-1-50901666-2, pp: 1086-1089.
Kazemian, M.E., S. Ebrahimi-Nejadc and M. Jaafariana, 2017. Experimental investigation of energy consumption and performance of reverse osmosis desalination using design of experiments method. IJE Intl. J. Eng. Trans. A. Basics, 31: 79-87.

Lira, J.L., 2010. [Digital treatment of multispectral images]. Master Thesis, Universidad Nacional Autonoma de Mexico, Mexico City, Mexico. (In Spanish)

Lopez, A.F.J., M.C.P. Pelayo and A.R. Forero, 2015. [Teaching images processing in engineering using python (In Spanish)]. VAEP. RITA., 3: 179-186.

Mery, D., 2004. [Computer Vision]. Pontificia Universidad Catolica de Chile, Santiago, Chile, Pages: 146 (In Spanish).

Mizutani, N., 1993. Real time monitoring of turbid water by using video camera, personal computer and image processing. Intl. Arch. Photogram. Remote Sens., 29: 520-520.

Polvorinos, A.J., M. Forteza and M.J. Hernandez, 2001. [Monitoring of hydraulic parameters of the Aracena reservoir through Landsat images (In Spanish)]. Remote Sens. Environ. Global Change, 1: 334-337.

Rebaza, J.V., 2007. [Edge detection using the canny algorithm]. Master Thesis, National University of Trujillo, Trujillo, Peru. (In Spanish)

Rodriguez, J.R., 2014. [Validation of turbidity and ph instrumental techniques for the treatment of drinking water in the treatment plant empocabal. esp-eice (holy rose of cabal)]. Master Thesis, Universidad Tecnologica de Pereira, Pereira, Colombia. (In Spanish)

Sultana, N., M.I. Hassana, N. Ridzuana, Z. Ibrahima and C.F. Soonc, 2018. Fabrication of gelatin scaffolds using thermally induced phase separation technique. Intl. J. Eng. Trans. B. Appl., 31: 1302-1307.

Vargas, J., F. Ramirez and J. Hernandez, 2018. Electroflocculation system based on power electronics that contributes to the treatment of contaminated water. Intl. J. Appl. Eng. Res., 13: 9480-9484.

Zain, N.M., E.N. Roslin and M.A. Ahamat, 2017. Effectiveness of warm water treatment on weld bonding performances of 6061 Aluminium Alloy. Intl. J. Appl. Eng. Res., 12: 14677-14686. 